\title{
A Remarkable Chord Iterative Method for Roots of Uncertain Multiplicity
}

\author{
I. Fried \\ Department of Mathematics, Boston University, Boston, MA, USA \\ Email: if@math.bu.edu
}

Received 23 May 2016; accepted 8 July 2016; published 11 July 2016

Copyright (C) 2016 by author and Scientific Research Publishing Inc.

This work is licensed under the Creative Commons Attribution International License (CC BY).

http://creativecommons.org/licenses/by/4.0/

(c) (i) Open Access

\begin{abstract}
In this note we at first briefly review iterative methods for effectively approaching a root of an unknown multiplicity. We describe a first order, then a second order estimate for the multiplicity index $m$ of the approached root. Next we present a second order, two-step method for iteratively nearing a root of an unknown multiplicity. Subsequently, we introduce a novel chord, or a twostep method, not requiring beforehand knowledge of the multiplicity index $m$ of the sought root, nor requiring higher order derivatives of the equilibrium function, which is quadratically convergent for any $m \leq 4$, and then reverts to superlinear.
\end{abstract}

\section{Keywords}

Iterative Methods, Unknown Root Multiplicity, Two-Step Methods

\section{Introduction}

The multiplicity index $m$ of root $x=a, f(a)=0$ of equilibrium function $f(x)$ may be a well latent property of the root, not cursorily revealed, nor readily available, yet this multiplicity can profoundly affect the behavior of the iterative approach [1]-[3] to the root. In this note, we briefly review the iterative methods [4]-[8] for approaching a root of an unknown multiplicity, and present a first oder [9] as well as a second order estimate for the multiplicity index $m$ of the approached root. Then we present a novel chord, or a two-step method, not requiring beforehand knowledge of $m$, nor requiring the higher derivatives of the equilibrium function, which is quadratically convergent for any $m \leq 4$, and then reverts to superlinear.

\section{Assumed Nature of the Equilibrium Function}

We assume that near root $a, f(a)=0$, function $f(x)$ has the power series representation 


$$
f(x)=(x-a)^{m}\left(A+B(x-a)+C(x-a)^{2}+\cdots\right), A \neq 0, m \geq 1
$$

where $m$ is the multiplicity index of root $a$, and where $A, B, C$, etc. are, for $m=1$, the coefficients

$$
A=f^{\prime}(a), B=\frac{1}{2 !} f^{\prime \prime}(a), C=\frac{1}{3 !} f^{\prime \prime \prime}(a)
$$

and so on.

\section{The Newton-Raphson Method}

The Newton-Raphson method

$$
x_{1}=x_{0}-u_{0}, u=\frac{f(x)}{f^{\prime}(x)}
$$

is quadratic

$$
x_{1}-a=\frac{B}{A}\left(x_{0}-a\right)^{2}+\frac{2\left(B^{2}+A C\right)}{A^{2}}\left(x_{0}-a\right)^{3}+O\left(\left(x_{0}-a\right)^{4}\right)
$$

if $m=1$. However, if $m>1$, the method declines to mere linear

$$
x_{1}-a=\frac{m-1}{m}\left(x_{0}-a\right)+\frac{B}{m^{2} A}\left(x_{0}-a\right)^{2}+O\left(\left(x_{0}-a\right)^{3}\right) .
$$

See also [10].

\section{Extrapolation to the Limit}

Let $x_{0}, x_{1}=x_{0}-u_{0}, x_{2}=x_{1}-u_{1}$ be already near root $a$. Then, if $m=1$

$$
x_{1}-a=\frac{B}{A}\left(x_{0}-a\right)^{2} \text { and } x_{2}-a=\frac{B}{A}\left(x_{1}-a\right)^{2}
$$

nearly. Eliminating $B / A$ from the two equations we obtain

$$
\left(-2 x_{0}+3 x_{1}-x_{2}\right) a^{2}+\left(x_{0}^{2}-3 x_{1}^{2}+2 x_{0} x_{2}\right) a+\left(x_{1}^{3}-x_{0}^{2} x_{2}\right)=0
$$

which we solve for an approximate $a$, as

$$
x_{3}=a=x_{0}-\frac{3+\sqrt{1+4 \rho}}{2(2-\rho)} u_{0}
$$

where

$$
\rho=u_{1} / u_{0}=\frac{B}{A}\left(x_{0}-a\right)+O\left(\left(x_{0}-a\right)^{2}\right) .
$$

The square root in Equation (8) may be approximated as

$$
\sqrt{1+4 \rho}=1+2 \rho-2 \rho^{2}+4 \rho^{3}-10 \rho^{4}+28 \rho^{5}-84 \rho^{6} \pm \cdots
$$

and for this extrapolated $x_{3}$ of Equation (8) we have

$$
x_{3}-a=\frac{2 B^{2}\left(B^{2}-A C\right)}{A^{4}}\left(x_{0}-a\right)^{5}+O\left(\left(x_{0}-a\right)^{6}\right) .
$$

For example, for $f(x)=x+x^{2}+x^{3}$, and starting with $x_{0}=0.2$, we compute $x_{1}=0.0368, x_{2}=0.0135$; and then from Equation (8), $x_{3}=0.000112$. Another such cycle starting with $x_{0}=x_{3}$ produces a next $x_{3}=-1.36 \times 10^{-20}$. 


\section{Always Quadratic Newton-Raphson Method}

The modified Newton-Raphson method

$$
x_{1}=x_{0}-m u_{0}=x_{0}-m \frac{f_{0}}{f_{0}^{\prime}}
$$

converges quadratically to a root of any multiplicity $m$

$$
x_{1}-a=\frac{1}{m} \frac{B}{A}\left(x_{0}-a\right)^{2}-\frac{1}{m^{2}}\left((m+1) \frac{B^{2}}{A^{2}}-2 m \frac{C}{A}\right)\left(x_{0}-a\right)^{3}+O\left(\left(x_{0}-a\right)^{4}\right) .
$$

But for this we need to know $m$.

By Equation (1) we readily deduce that, for any $x$

$$
\mu=\frac{1}{u^{\prime}}=\frac{f^{\prime 2}}{f^{\prime 2}-f f^{\prime \prime}}=m+\frac{2 B}{A}(x-a)+\frac{B^{2}-3 B^{2} m+6 A C m}{A^{2} m}(x-a)^{2}+O\left((x-a)^{3}\right)
$$

obtained at the price of a second derivative. For finite-difference approximations of the needed derivatives see [11]-[13]. Using $\mu$ in Equation (14) for $m$ in Equation (12) we obtain the method

$$
x_{1}=x_{0}-\frac{f_{0} f_{0}^{\prime}}{f_{0}^{\prime 2}-f_{0} f_{0}^{\prime \prime}}
$$

which is quadratic for any, provided, $m$

$$
x_{1}-a=-\frac{1}{m} \frac{B}{A}\left(x_{0}-a\right)^{2}+O\left(\left(x_{0}-a\right)^{3}\right) .
$$

The method of Equation (15) is also obtained by applying Newton's method not to $f$, but rather to $u=f / f^{\prime}$. For $f(x)=x^{m}(3+x)$, we obtain by the method of Equation (15) that requires not only $f^{\prime}$ but also $f^{\prime \prime}$, starting with $x_{0}=1$.

For $m=1$

$$
x=\left\{1,-0.176,-0.012,-4.6 \times 10^{-5},-6.98 \times 10^{-10},-1.63 \times 10^{-19}\right\} .
$$

For $m=7$

$$
x=\left\{1,-0.027,-3.4 \times 10^{-5},-5.6 \times 10^{-11},-1.47 \times 10^{-22}\right\} .
$$

Equation (15) may be written as

$$
x_{1}=x_{0}-\frac{1}{1-z_{0}} \frac{f_{0}}{f_{0}^{\prime}}, z_{0}=\frac{f_{0} f_{0}^{\prime \prime}}{f_{0}^{\prime 2}}
$$

and it is of interest to know that

$$
z_{0}=\frac{f_{0} f_{0}^{\prime \prime}}{f_{0}^{\prime 2}}=\frac{m-1}{m}+\frac{2}{m^{2}} \frac{B}{A}(x-a)+O\left((x-a)^{2}\right) .
$$

For the price of a third derivative we may have the quadratic approximation

$$
\mu=\frac{u^{\prime}}{u^{\prime 2}-u u^{\prime \prime}}=\frac{f^{\prime 2}\left(f^{\prime 2}-f f^{\prime \prime}\right)}{f^{\prime 4}-f f^{\prime 2} f^{\prime \prime}-f^{2} f^{\prime \prime 2}+f^{2} f^{\prime \prime \prime \prime}}=\frac{m+B^{2}+3 B^{2} m-6 A C m}{A^{2} m}\left(x_{0}-a\right)^{2}+\cdots
$$

\section{An Erroneous m}

The method 


$$
x_{1}=x_{0}-m(1+\epsilon) u_{0}
$$

produces the superlinear

$$
x_{1}-a=-\epsilon\left(x_{0}-a\right)+\frac{B(1+\epsilon)}{A m}\left(x_{0}-a\right)^{2}+O\left(\left(x_{0}-a\right)^{3}\right)
$$

and if $\epsilon>0$, convergence is alternating.

\section{Estimation of the Leading Term}

We readily have that

$$
-\frac{1}{2} m^{2} u^{\prime \prime}=-\frac{1}{2} m^{2} \frac{-f^{\prime 2} f^{\prime \prime}+2 f f^{\prime \prime 2}-f f f^{\prime \prime \prime}}{f^{\prime 3}}=\frac{B}{A}-\left(\frac{3(1+m)}{m}\left(\frac{B}{A}\right)^{2}-6 \frac{C}{A}\right)(x-a) .
$$

For example, for $f=x+10 x^{2}$, we compute using Equation (23) the $B / A$ approximations as depending on the $\operatorname{chosen} x$

$$
\{x, B / A\}=\left\{10^{-2}, 5.79\right\},\left\{10^{-3}, 9.42\right\},\left\{10^{-4}, 9.94\right\},\left\{10^{-5}, 9.994\right\} .
$$

\section{An Elementary Discrete Two-Step Newton Method for Roots of Any Multiplicity} If

$$
x_{0}, x_{1}=x_{0}-u_{0}, x_{2}=x_{1}-u_{1}, u=\frac{f}{f^{\prime}}
$$

are already close to root $a$ of multiplicity $m>1$, then according to Equation (5)

$$
x_{1}-a=\left(1-\frac{1}{m}\right)\left(x_{0}-a\right), \text { and } x_{2}-a=\left(1-\frac{1}{m}\right)\left(x_{1}-a\right)
$$

nearly, from which we extract the approximation

$$
a=\frac{x_{1}^{2}-x_{0} x_{2}}{-x_{0}+2 x_{1}-x_{2}}=x_{0}-\frac{u_{0}}{u_{0}-u_{1}} u_{0}=x_{1}-\frac{u_{0}}{u_{0}-u_{1}} u_{1} .
$$

Setting $a$ back into Equation (26) yields

$$
\mu=\frac{x_{1}-x_{0}}{u_{1}-u_{0}}=\frac{1}{1-\rho}, \rho=\frac{u_{1}}{u_{0}}
$$

and the two-step method

$$
\mu_{0}=\mu_{0}, u_{0}=\frac{f_{0}}{f_{0}^{\prime}}, x_{1}=x_{0}-\mu_{0} u_{0}, u_{1}=\frac{f_{1}}{f_{1}^{\prime}}, \mu_{1}=\frac{x_{1}-x_{0}}{u_{1}-u_{0}}=\frac{1}{1-\rho}, \rho=\frac{u_{1}}{u_{0}}, x_{2}=x_{1}-\mu_{1} u_{1}
$$

where $\mu$ in Equation (28) is seen to be but the finite-difference approximation of $\mu=\mathrm{d} x / \mathrm{d} u$ in Equation (14).

For example, for $f(x)=x^{3}+x^{4}$, and starting with $x_{0}=1, \mu_{0}=1$, we compute by Equation (29), the successive approximations

$$
\begin{gathered}
x_{0}=1, \mu_{0}=1, x_{1}=0.71, \mu_{1}=3.72, x_{2}=-6.4 \times 10^{-2} \\
x_{0}=-0.064, \mu_{0}=3.72, x_{1}=0.018, \mu_{1}=2.95, x_{2}=4 \times 10^{-4} \\
x_{0}=4 \times 10^{-4}, \mu_{0}=2.95, x_{1}=6.9 \times 10^{-6}, \mu_{1}=3.0004, x_{2}=-9.3 \times 10^{-10}
\end{gathered}
$$




$$
x_{0}=-9.3 \times 10^{-10}, \mu_{0}=3.0004, x_{1}=1.26 \times 10^{-13}, \mu_{1}=3, x_{2}=3.9 \times 10^{-23} \text {. }
$$

Generally, starting with

$$
\mu_{0}=m+\epsilon_{1}, x_{0}=a+\epsilon_{2}
$$

we have from the method of Equation (29) that

$$
\mu_{1}=m+\frac{B}{A}\left(1-\frac{\epsilon_{1}}{m}\right) \epsilon_{2}+O\left(\epsilon_{2}^{2}\right), x_{2}=a+\frac{B}{A m^{2}} \epsilon_{1} \epsilon_{2}^{2}+O\left(\epsilon_{2}^{3}\right)
$$

The repeated classical Newton's method, $x_{1}=x_{0}-f_{0} / f_{0}^{\prime}, x_{2}=x_{1}-f_{1} / f_{1}^{\prime}$, we recall, is only linear if $m>1$

$$
x_{2}-a=\left(1-\frac{1}{m}\right)^{2}\left(x_{0}-a\right)+\frac{(2 m-1)(m-1)}{m^{4}} \frac{B}{A}\left(x_{0}-a\right)^{2}+O\left(\left(x_{0}-a\right)^{3}\right) \text {. }
$$

See also [14] [15].

\section{Derivation of the Chord Method}

It is a rational two step method of the form

$$
x_{2}=x_{1}+\left(x_{1}-x_{0}\right) \frac{f_{1}+P f_{0}}{Q f_{1}+R f_{0}}, x_{1}=x_{0}+k \frac{f_{0}}{f_{0}^{\prime}}, f_{0}=f\left(x_{0}\right), f_{1}=f\left(x_{1}\right) .
$$

With

$$
P=\frac{6+11 k+6 k^{2}+k^{3}}{-6+4 k}, Q=\frac{9-2 k}{-3+2 k}, R=\frac{18+14 k+5 k^{2}+k^{3}}{6-4 k}
$$

the method is quadratic for $m=1, m=2$ and $m=3$. In fact;

For $m=1$

$$
x_{2}-a=-\frac{B}{A} \frac{9+7 k}{9+k}\left(x_{0}-a\right)^{2}+O\left(\left(x_{0}-a\right)^{3}\right) \text {. }
$$

For $m=2$

$$
x_{2}-a=-\frac{B}{2 A} \frac{9+7 k+k^{2}}{9+4 k}\left(x_{0}-a\right)^{2}+O\left(\left(x_{0}-a\right)^{3}\right) .
$$

For $m=3$

$$
x_{2}-a=-\frac{B}{3 A} \frac{81+63 k+14 k^{2}}{81+45 k+4 k^{2}}\left(x_{0}-a\right)^{2}+O\left(\left(x_{0}-a\right)^{3}\right) .
$$

For $m=4$ the method produces

$$
x_{2}-a=\frac{(k-2) k^{2}}{576+352 k+46 k^{2}+4 k^{3}}\left(x_{0}-a\right)+O\left(\left(x_{0}-a\right)^{2}\right)
$$

and for $k=2$ the method is quadratic for $m=4$ as well.

According to Equation (36a), if $m=1, k=-9 / 7$, then the method is higher than quadratic.

\section{The Method is Further Superlinear}

For $k=2$ we have:

For $m=1$

$$
x_{2}-a=-\frac{23 B}{11 A}\left(x_{0}-a\right)^{2}+\frac{914 B^{2}-1628 A C}{121 A^{2}}\left(x_{0}-a\right)^{3}+O\left(\left(x_{0}-a\right)^{4}\right) .
$$


For $m=2$

$$
x_{2}-a=-\frac{27 B}{34 A}\left(x_{0}-a\right)^{2}+\frac{1277 B^{2}-2414 A C}{578 A^{2}}\left(x_{0}-a\right)^{3}+O\left(\left(x_{0}-a\right)^{4}\right) .
$$

For $m=3$

$$
x_{2}-a=-\frac{263 B}{561 A}\left(x_{0}-a\right)^{2}+\frac{370334 B^{2}-715836 A C}{314721 A^{2}}\left(x_{0}-a\right)^{3}+O\left(\left(x_{0}-a\right)^{4}\right) .
$$

For $m=4$

$$
x_{2}-a=-\frac{245 B}{748 A}\left(x_{0}-a\right)^{2}+\frac{435571 B^{2}-851224 A C}{559504 A^{2}}\left(x_{0}-a\right)^{3}+O\left(\left(x_{0}-a\right)^{4}\right) .
$$

For $m=5$

$$
x_{2}-a=\frac{1}{9871.9}\left(x_{0}-a\right)-\frac{1}{4} \frac{B}{A}\left(x_{0}-a\right)^{2}+O\left(x_{0}-a\right)^{3} \text {. }
$$

For $m=7$

$$
x_{2}-a=\frac{1}{1657}\left(x_{0}-a\right)-\frac{B}{5.95 A}\left(x_{0}-a\right)^{2}+O\left(x_{0}-a\right)^{3} .
$$

For $m=9$

$$
x_{2}-a=\frac{1}{718}\left(x_{0}-a\right)-\frac{B}{7.94 A}\left(x_{0}-a\right)^{2}+O\left(x_{0}-a\right)^{3} \text {. }
$$

For $m=11$

$$
x_{2}-a=\frac{1}{423.3}\left(x_{0}-a\right)-\frac{B}{9.97 A}\left(x_{0}-a\right)^{2}+O\left(x_{0}-a\right)^{3} .
$$

For $m=17$

$$
x_{2}-a=\frac{1}{171.4}\left(x_{0}-a\right)-\frac{B}{16.2 A}\left(x_{0}-a\right)^{2}+O\left(x_{0}-a\right)^{3} .
$$

For $m=27$

$$
x_{2}-a=\frac{1}{81}\left(x_{0}-a\right)-\frac{B}{27 A}\left(x_{0}-a\right)^{2}+O\left(x_{0}-a\right)^{3}
$$

\section{Lowering the Value of $\boldsymbol{k}$}

We leave $k$ in $x_{1}=x_{0}+k f_{0} / f_{0}^{\prime}$ of Equation (34), free, and have by power series expansion, for multiplicity index $m=5$, for $f(x)$ in Equation (1), that

$$
x_{2}-a=\frac{2 k^{2}}{5} \frac{-125+55 k+4 k^{2}}{5625+3625 k+550 k^{2}+82 k^{3}+4 k^{4}}\left(x_{0}-a\right)+O\left(\left(x_{0}-a\right)^{2}\right) .
$$

The linear term in the above expansion is annulled with

$$
-125+55 k+4 k^{2}=0, k=1.9859043 .
$$

We do this for higher values of $m$ and find that

$$
\{m, k\}=\{4,2\},\{5,1.9859043\},\{7,1.9689621\},\{9,1.9591333\},\{11,1.9527133\} .
$$

We try $k=1.95$, and get 
For $m=1$

$$
x_{2}-a=-8.9 \times 10^{-16}\left(x_{0}-a\right)-\frac{2.07 B}{A}\left(x_{0}-a\right)^{2}+O\left(\left(x_{0}-a\right)^{3}\right) .
$$

For $m=2$

$$
x_{2}-a=-6.6 \times 10^{-16}\left(x_{0}-a\right)-\frac{0.787 B}{A}\left(x_{0}-a\right)^{2}+O\left(\left(x_{0}-a\right)^{3}\right) .
$$

For $m=3$

$$
x_{2}-a=-4.4 \times 10^{-16}\left(x_{0}-a\right)-\frac{0.466 B}{A}\left(x_{0}-a\right)^{2}+O\left(\left(x_{0}-a\right)^{3}\right) .
$$

For $m=4$

$$
x_{2}-a=-17712\left(x_{0}-a\right)-\frac{0.326 B}{A}\left(x_{0}-a\right)^{2}+O\left(\left(x_{0}-a\right)^{3}\right) .
$$

For $m=5$

$$
x_{2}-a=-13999\left(x_{0}-a\right)-\frac{0.249 B}{A}\left(x_{0}-a\right)^{2}+O\left(\left(x_{0}-a\right)^{3}\right) .
$$

For $m=7$

$$
x_{2}-a=-12799\left(x_{0}-a\right)-\frac{0.168 B}{A}\left(x_{0}-a\right)^{2}+O\left(\left(x_{0}-a\right)^{3}\right) .
$$

For $m=9$

$$
x_{2}-a=-13315\left(x_{0}-a\right)-\frac{0.127 B}{A}\left(x_{0}-a\right)^{2}+O\left(\left(x_{0}-a\right)^{3}\right) .
$$

For $m=11$

$$
x_{2}-a=-17608\left(x_{0}-a\right)-\frac{0.101 B}{A}\left(x_{0}-a\right)^{2}+O\left(\left(x_{0}-a\right)^{3}\right) .
$$

For $m=17$

$$
x_{2}-a=11312\left(x_{0}-a\right)-\frac{0.063 B}{A}\left(x_{0}-a\right)^{2}+O\left(\left(x_{0}-a\right)^{3}\right) .
$$

For $m=27$

$$
x_{2}-a=1358\left(x_{0}-a\right)-\frac{0.038 B}{A}\left(x_{0}-a\right)^{2}+O\left(\left(x_{0}-a\right)^{3}\right) .
$$

For $m=37$

$$
x_{2}-a=1198\left(x_{0}-a\right)-\frac{0.027 B}{A}\left(x_{0}-a\right)^{2}+O\left(\left(x_{0}-a\right)^{3}\right) .
$$

The general form of the linear part of $x_{2}-a$ in Equations (42) is of the form $c(m)\left(x_{0}-a\right)$ with a constant $c(m)$ that is small if multiplicity index $m$ is not much above 5. For instance, $c(11)=-1 / 7608$, meaning that at each iteration the error $x_{2}-a$ is reduced by this factor. Such convergence behavior we term superlinear. More concretely, for $f(x)=x^{m}(3+x)$, we obtain by the above method, using $k=1.95$, starting with $x_{0}=1$.

For $m=1$

$$
x=\left\{1,-0.26,-0.066,-7.2 \times 10^{-6},-3.6 \times 10^{-11},-9 \times 10^{-22}\right\}
$$


For $m=3$

$$
x=\left\{1,-0.76,-9.6 \times 10^{-4},-1.44 \times 10^{-7},-3.2 \times 10^{-15}, 7.9 \times 10^{-31}\right\}
$$

For $m=7$

$$
x=\left\{1,-0.03,-4.1 \times 10^{-5}, 1.47 \times 10^{-8},-5.2 \times 10^{-12}, 1.88 \times 10^{-15},-6.7 \times 10^{-19}, 2.4 \times 10^{-22}\right\} .
$$

\section{Conclusions}

The paper is predicated on the realistic assumption that the multiplicity index $m$ of the iteratively targeted root is unknown. We conclude that if one prefers to shun second order derivatives, then the quadratic two-step method of Equation (29), that provides also ever better approximations for the multiplicity index $m$ of the approached root, is a practically appealing alternative.

Otherwise, one may use the rational two-step method of Equation (34) with a constant $k$ that is only slightly less than 2. Thus stating the method becomes superlinear, albeit, of a reduced speed of convergence for highly elevated root multiplicities. For the sake of brevity, the present paper does not explore the possibility of estimating the multiplicity index $m$ of the sought root by the method of Equation (29), then applying this estimate to the choice of an optimal $k$ in the method of Equations (34) and (35).

\section{References}

[1] Householder, A.S. (1970) The Numerical Treatment of a Single Nonlinear Equation. McGraw-Hill, New-York.

[2] Traub, J.F. (1977) Iterative Methods for the Solution of Equations. Chelsea Publishing Company, New York.

[3] Ostrowski, A. (1960) Solution of Equations and Systems of Equations. Academic Press, New York.

[4] Hansen, E. and Patrick, M.A. (1977) Family of Root Finding Methods. Numerische Mathematik, 27, $257-269$. http://dx.doi.org/10.1007/BF01396176

[5] Petkovic, M.S., Petkovic, L.D. and Dzunic, J. (2010) Accelerating Generators of Iterative Methods for Finding Multiple Roots of Nonlinear Equations. Computers and Mathematics with Applications, 59, 2784-2793. http://dx.doi.org/10.1016/j.camwa.2010.01.048

[6] Neta, B. and Johnson, A.N. (2008) High-Order Nonlinear Solver for Multiple Roots. Computers and Mathematics with Application, 55, 2012-2017. http://dx.doi.org/10.1016/j.camwa.2007.09.001

[7] Fried, I. (2013) High-Order Iterative Bracketing Methods. International Journal for Numerical Methods in Engineering, 94, 708-714. http://dx.doi.org/10.1002/nme.4467

[8] King, R.F. (1977) A Secant Method for Multiple Roots. BIT, 17, 321-328. http://dx.doi.org/10.1007/BF01932152

[9] Lagouanelle, J.L. (1966) Sur Une Metode de Calcul de 1'Ordre de Multiplicite des Zeros d'Un Polynome. Comptes Rendus de l'Académie des Sciences, 262, 626-627.

[10] Rall, L.B. (1966) Convergence of the Newton Process to Multiple Solutions. Numerische Mathematik, 9, 23-37. http://dx.doi.org/10.1007/BF02165226

[11] Soleymani, F. (2012) Optimized Steffensen-Type Methods with Eighth-Order Convergence and High Efficiency Index. International Journal of Mathematics and Mathematical Sciences, 2012, 1-18. http://dx.doi.org/10.1155/2012/932420

[12] Sharma, J.R. (2005) A Composite Third Order Newton-Steffensen Method for Solving Nonlinear Equations. Applied Mathematics and Computation, 169, 242-246. http://dx.doi.org/10.1016/j.amc.2004.10.040

[13] Esser, H. (1975) Eine Stets Quadratisch Konvergente Modifikation des Steffensen Verfahrens. Computing, 14, 367369. http://dx.doi.org/10.1007/BF02253547

[14] Dong, C. (1987) A Family of Multipoint Iterative Functions for Finding Multiple Roots of Equations. International Journal of Computer Mathematics, 21, 363-367. http://dx.doi.org/10.1080/00207168708803576

[15] Thukral, R. (2013) Introduction to Higher-Order Iterative Methods for Finding Multiple Roots of Nonlinear Equations. Journal of Mathematics, 2013, 1-3. http://dx.doi.org/10.1155/2013/404635 


\section{Submit or recommend next manuscript to SCIRP and we will provide best service for you:}

Accepting pre-submission inquiries through Email, Facebook, Linkedin, Twitter, etc A wide selection of journals (inclusive of 9 subjects, more than 200 journals)

Providing a 24-hour high-quality service

User-friendly online submission system

Fair and swift peer-review system

Efficient typesetting and proofreading procedure

Display of the result of downloads and visits, as well as the number of cited articles

Maximum dissemination of your research work

Submit your manuscript at: http://papersubmission.scirp.org/ 